\title{
miR-495 enhances the efficacy of radiotherapy by targeting GRP78 to regulate EMT in nasopharyngeal carcinoma cells
}

\author{
XUEPING FENG ${ }^{1}$, WUWU LV ${ }^{1}$, SHUANGLIAN WANG ${ }^{1}$ and QIAN HE $^{2}$ \\ ${ }^{1}$ Institute of Medical Sciences, Xiangya Hospital, Central South University, Changsha, Hunan 410078; \\ ${ }^{2}$ Department of Radiation Oncology, Hunan Cancer Hospital and The Affiliated Cancer Hospital of \\ Xiangya School of Medicine, Central South University, Changsha, Hunan 410013, P.R. China
}

Received June 29, 2017; Accepted June 7, 2018

DOI: $10.3892 / o r .2018 .6538$

\begin{abstract}
Glucose-regulated protein 78 (GRP78) was revealed to be associated with the radioresistance of nasopharyngeal carcinoma (NPC) in our previous study. GRP78 is a highly expressed cell surface protein, and holds great promise as a cancer specific target. Its expression may be impacted by the regulation of miRNAs, which may be involved in the radioresistance of NPC. A better understanding of the mechanisms of radioresistance may generate new targets of therapy for NPC patients. The present study was designed to investigate the effect of microRNA targeting GRP78 on the radiosensitivity of NPC. First, we used miRWalk software to predict miRNAs that may interact with GRP78. Subsequently, analysis of miR-495 and GRP78 expression was performed in the primary tissues of 92 NPC tissues and cell lines by immunohistochemistry and real-time PCR and the results revealed that miR-495 expression was lower in radioresistant NPC tissues in comparison to chronic rhinitis tissues, and also lower in radioresistant $5-8 \mathrm{~F}$ cells $(5-8 \mathrm{~F}-\mathrm{IR})$ in comparison to its parental $5-8 \mathrm{~F}$ cells. Notably, we observed an inverse association between the expression miR-495 and GRP78. Our bioinformatics analysis led to the identification of miR-495 as the optimal miRNA interacting with GRP78 mRNA.
\end{abstract}

Correspondence to: Dr Xueping Feng, Institute of Medical Sciences, Xiangya Hospital, Central South University, 87 Xiangya Road, Kaifu, Changsha, Hunan 410078, P.R. China

E-mail: fengxuepingping@tom.com

Dr Qian He, Department of Radiation Oncology, Hunan Cancer Hospital and The Affiliated Cancer Hospital of Xiangya School of Medicine, Central South University, 283 Tongzipo Road, Yuelu, Changsha, Hunan 410013, P.R. China

E-mail: zhouhe0121@163.com

Abbreviations: EMT, epithelial-mesenchymal transition; UTR, untranslated region; miRNA, microRNA; NPC, nasopharyngeal carcinoma; GRP78, glucose-regulated protein 78; RT-qPCR, real-time fluorescence quantitative polymerase chain reaction

Key words: miR-495, nasopharyngeal carcinoma, radioresistance, glucose-regulated protein 78 , epithelial-mesenchymal transition
Furthermore, miR-495 targeting the 3'untranslated region (UTR) of GRP78 was detected by a Dual-Glo Luciferase Assay system. Finally, we observed that miR-495 inhibition led to a significant increase in the radioresistance of $5-8 \mathrm{~F}$ cells and higher GRP78 expression, which may be involved in epithelial-mesenchymal transition (EMT) phenotype. miR-495 targeted the 3'UTR of GRP78 and contributed to the efficacy of radiation therapy in NPC.

\section{Introduction}

Nasopharyngeal carcinoma (NPC) is a common head and neck tumor with mortality rates of $15-50$ per 100,000 people in Southeast Asia, especially in southern China (1). Since $>90 \%$ of the NPC cases are poorly distinguished squamous cell carcinomas, they display radiosensitivity, and thus radical radiotherapy is the first choice of primary treatment (2). However, radiotherapy still has many obstacles to overcome, like radioresistance (3). To overcome this critical issue and correctly predict the required radiation levels for individualized treatment, the role of many radiosensitive biomarkers has been analyzed, with the hope that some of them will help to predict clinical radiosensitivity, and improve the therapeutic efficacy along with revealing the molecular mechanism of NPC radioresistance. We determined that glucose-regulated protein 78 (GRP78) was a radioresistant protein of NPC in our previous study (4).

GRP78, a multifunctional protein folding chaperone and co-receptor is highly expressed on the cell surface, and holds promise for cancer-specific targeting. Its role has specifically been studied in colon and breast cancer $(5,6)$. It has also been revealed to play an analeptic role in invasive procedures and possibly an anti-invasive target for treating hepatocellular carcinoma (7). GRP78 expression is induced in multiple cancers as a result of endoplasmic reticulum stress induced either by acidosis, glucose deprivation or severe hypoxia. Chemotherapeutic drugs and radiation have also been revealed to induce GRP78 protein expression, which in turn confers resistance in the tumor cells against these agents (8). In addition, the changes of GRP78 expression have also been revealed to play key functions in the development and progression of malignant tumors (9). GRP78 has been revealed to be a therapeutic target of NPC (10). 
Our previous efforts in identifying the proteins responsible for NPC radioresistance indicated GRP78 as a potential player for predicting NPC response to radiotherapy (4). However, the mechanism of its functional/expression regulation in NPC following radiotherapy remains unclear.

MicroRNAs (miRNAs) are small non-coding RNAs (20-24 nucleotides in length) which post-transcriptionally modulate gene expression through negative regulation of the stability or translational efficiency of their target mRNAs $(11,12)$. These effects are obtained by miRNA binding to the 3 -untranslated region (3'UTR) of their target mRNAs, which can reduce the expression of the associated protein.

Thus, in the present study, we investigated the mechanism of GRP78 regulation by first performing bioinformatics analysis to identify putative miRNA targeting GRP78. Our initial analysis led to the identification of miR-495 as the miRNA binding to GRP78 mRNA.

In the present study, we examined the impact of miR-495-mediated targeting of cell surface associated GRP78, and its effects on cell survival and growth when combined with X-ray treatment. In the present study, miR-495 mimics suppressed cell proliferation and induced cell death. GRP78 was targeted by miR-495 and miR-495 mimics also enhanced the efficacy of radiotherapy. Subsequently, we analyzed the mechanism of miR-495-mediated regulation of GRP78 expression and its role in regulating the radiosensitivity of NPC cells.

\section{Materials and methods}

Patients and tissue specimens. A total of 92 NPC patients (52 males and 40 females) were included in the present study with a median age of 48 years (range, $12-85$ years) and 30 patients (18 males and 12 females) with chronic rhinitis were enrolled as controls (median age 44 years; range, 20-73 years). The information of all NPC patients that underwent curative-intent radiotherapy treatment between August 2012 and July 2014 was reviewed and it was observed that the overall radiation dose of 60-70 Gy was administered to each patient using a modified type of linear accelerator (Xiangya Hospital of Central South University, Changsha, China). Based on this therapy, 39 patients were diagnosed as radioresistant, while 53 were radiosensitive. The patients were characterized as radioresistant, if they exhibited persisting disease (incomplete regression of tumors) after 6 weeks of completing radiotherapy, or those with recurrent disease at the nasopharynx and/or neck nodes after 2 months post-radiotherapy (13). Similarly, the patients were characterized as radiosensitive, if they did not display any local residual lesions after 6 weeks or any recurrence of symptoms after 2 months of completing radiotherapy (13). NPC tissue biopsies (pre-therapy) were obtained after informed consent was received from all participants and were used for immunohistochemical (IHC) staining. All the patient specimens were separately reviewed by two experienced pathologists and the diagnoses were confirmed by histopathological examination. The diagnoses were based on the 1978 WHO classification (14). This study was certified by the Ethics Committee of Xiangya School of Medicine (Central South University, Changsha, China).

Cell culture. NPC cell line 5-8F was purchased from the Cell Bank of Type Culture Collection of the Chinese Academy of
Sciences (Shanghai, China). 5-8F was seeded in a 6-cm plate ( $1 \times 10^{5}$ cells). The radioresistant NPC cell line 5-8F-IR was derived from NPC cell line 5-8F. 5-8F-IR was established by our laboratory (Xiangya Hospital of Central South University). These cells were cultured in RPMI-1640 medium (Gibco; Thermo Fisher Scientific, Inc., Waltham, MA, USA), supplemented with 10\% fetal bovine serum (FBS; Hangzhou Sijiqing Biological Engineering Materials, Co., Ltd., Hangzhou, China) and incubated at $37^{\circ} \mathrm{C}$ in a $5 \% \mathrm{CO}_{2}$ atmosphere. After $24 \mathrm{~h}$ of culture, the radiation of the sublethal $10 \mathrm{~Gy}$ dose was delivered at room temperature at $300 \mathrm{cGy} / \mathrm{min}$ with a linear accelerator (2100EX; Varian Medical Systems, Inc., Palo Alto, CA, USA). Following treatment, the radioresistant cells that had survived produced the generation of the subclones 15 days later.

Other reagents. The IHC kit (S-P) and 3,3'-diaminobenzidine (DAB) developing solution were purchased from Fuzhou Maixin Biotech Co., Ltd., (Fuzhou, China). Mouse anti-human monoclonal antibodies to GRP78 (1:500; cat. no. sc-376768), E-cadherin (1:500; cat. no. sc-71007), N-cadherin (1:500; cat. no. sc-53488), vimentin (1:500; cat. no. c-80975) and $\beta$-actin (1:1,000; cat. no. sc-58673) were all purchased from Santa Cruz Biotechnology, Inc. (Dallas, TX, USA). Lipofectamine 2000 was purchased from Invitrogen (Thermo Fisher Scientific, Inc., Waltham, MA, USA) and the Transwell assay kit was procured from Corning Inc. (Corning, NY, USA).

Bioinformatics analysis. The prediction analysis of miRNAs binding to GRP78 mRNA was performed using miRWalk online program (http://zmf.umm.uni-heidelberg. $\mathrm{de} / \mathrm{apps} / \mathrm{zmf} / \mathrm{mirwalk} /$ predictedmirnagene.html). The predicted miRNAs with the highest binding scores were further identified using miRanda software (http://www. microrna.org/microrna/home.do). This software computes thermodynamic stability and sequence conservation scores.

Immunohistochemistry staining. IHC staining of NPC tissue specimens was performed using the previously described S-P IHC method (4). The cytoplasm staining intensity was scored by two pathologists as follows: No color, negative; pale yellow, weakly positive; brown, positive; and tan, strongly positive. Overall the percentage of tissue samples with positive expression was calculated by the following formula: (Total number of samples with weakly positive + positive + strongly positive staining/total number of samples evaluated) $\mathrm{x} 100$.

$R T-q P C R$ analysis. Total RNA was extracted from fresh NPC and chronic rhinitis tissue samples by homogenization in TRIzol reagent (Gibco; Thermo Fisher Scientific, Inc.). PCR amplification of miR-495 was carried out using a two-step method. The following primers were designed based on the precursor sequence of miR-495: Forward primer, 5'-UGG UACCUGAAAAGAAGUUG-3' and reverse, 5'-GCACUU CUUUUUCGGUAUCA-3'. For U6 gene amplification, the following primers were used: Forward primer, 5'-GCTTCG GCAGCACATATACTAAAAT-3' and reverse, 5'-CGCTTC ACGAATTTGCGTGTCAT-3'. All these primers were synthesized by Guangzhou RiboBio Co., Ltd. (Guangzhou, China), and the amplification reactions were carried out using a fluorescence RT-qPCR instrument, based on the manufacturer's 
Table I. Expression of GRP78 in NPC and chronic rhinitis tissues.

\begin{tabular}{|c|c|c|c|c|c|c|}
\hline & \multirow[b]{2}{*}{$\mathrm{N}$} & \multicolumn{3}{|c|}{ Scores of immunohistochemical staining } & \multirow[b]{2}{*}{$\chi^{2}$} & \multirow[b]{2}{*}{ P-value } \\
\hline & & Weak positive/negative $(0-2)$ & Positive (3-4) & Strong positive $(5-6)$ & & \\
\hline Chronic rhinitis & 30 & 26 & 3 & 1 & 30.621 & $0.000^{\mathrm{a}}$ \\
\hline NPC & 92 & 27 & 30 & 35 & & \\
\hline Radioresistant & 39 & 5 & 14 & 20 & 9.644 & $0.008^{\mathrm{b}}$ \\
\hline Radiosensitive & 53 & 22 & 16 & 15 & & \\
\hline
\end{tabular}

${ }^{a} \mathrm{P}<0.05$, chronic rhinitis vs. NPC; ${ }^{b} \mathrm{P}<0.05$, radioresistant vs. radiosensitive. Using $\chi^{2}$ test. NPC, nasopharyngeal carcinoma.

instructions. The following RT-qPCR reaction protocol was used: $2.5 \mu \mathrm{l}$ of dNTPs (2.5 mM each); $2.5 \mu \mathrm{l}$ of $10 \mathrm{X}$ PCR buffer; $1.5 \mu \mathrm{l}$ of $\mathrm{MgCl}_{2}$ (MW 95.21) solution; 1 unit of Taq polymerase; SYBR-Green I, final concentration $0.25 \mathrm{X} ; 1 \mu \mathrm{l}$ of forward primer and reverse primer (10 $\mu \mathrm{M}$ stock); $1 \mu \mathrm{l} \mathrm{cDNA}$; and water to a total volume of $25 \mu \mathrm{l}$. The U6 gene amplification reaction was as follows: $95^{\circ} \mathrm{C}$ for $5 \mathrm{~min} ; 35$ cycles $\left(95^{\circ} \mathrm{C}\right.$ for $10 \mathrm{sec} ; 59^{\circ} \mathrm{C}$ for $15 \mathrm{sec} ; 72^{\circ} \mathrm{C}$ for $20 \mathrm{sec}$; and $82^{\circ} \mathrm{C}$ for $5 \mathrm{sec}$ ). The miRNA reaction was performed as follows: $95^{\circ} \mathrm{C}$ for $15 \mathrm{~min}$; 40 cycles $\left(94^{\circ} \mathrm{C}\right.$ for $15 \mathrm{sec} ; 55^{\circ} \mathrm{C}$ for $30 \mathrm{sec}$; and $70^{\circ} \mathrm{C}$ for $\left.30 \mathrm{sec}\right)$.

Western blotting. To extract the total cellular protein, tissues or cells were incubated with pre-cooled RIPA lysis buffer (Thermo Fisher Scientific, Inc.), vortexed and then placed on ice for $30 \mathrm{~min}$. After centrifugation, the supernatants were removed and the protein concentrations were estimated using the Bradford method. The proteins were denatured by incubation for $5 \mathrm{~min}$ at $100^{\circ} \mathrm{C}$ and then the loading buffer was added. Subsequenlty, $20 \mu \mathrm{g}$ of the denatured proteins per lane were separated by $12 \%$ gel electrophoresis and transferred to polyvinylidene difluoride (PVDF) membranes. The membranes were blocked by incubating them in a blocking buffer containing $5 \%$ non-fat milk powder for $1-2 \mathrm{~h}$ and then washed and incubated with the appropriate primary antibody overnight at $4^{\circ} \mathrm{C}$. The primary antibodies were diluted as follows: GRP78 (1:500), E-cadherin (1:500), N-cadherin (1:500), vimentin (1:500) and $\beta$-actin (1:1,000). For detection enhanced chemiluminescence (ECL; Santa Cruz Biotechnology, Inc.) was used. The protein bands were analyzed using Band leader 3.0.

Construction of GRP78 3'UTR plasmids. The bioinformatics software predicted the binding between miR-495 and GRP78 mRNA. RT-PCR was used to amplify a sequence encompassing these 501 base pairs. The primers for amplification were designed as follows: GRP78_WT_forward, 5'-ACT GCTGTTTTCAGATGGAGGT-3' and reverse, 5'-CTAGGA GCCAGCTCAGATGC-3'; GRP78_mut_forward, 5'-TGC GGAGATCTATCTATCATGGC-3' and reverse, 5'-GGT GTCAGGCGATTCTGGTC-3'. The amplified fragments were cloned into the pmiR-RB-REPORT ${ }^{\mathrm{TM}}$ dual luciferase reporter vector (Guangzhou RiboBio Co., Ltd., Guangzhou, China). The hRluc vector was used to report fluorescence, and the 3'UTR of GRP78 was cloned downstream of the hRluc gene. The directly targeted region was determined by cloning the 3'UTR seed region and the mutated seed region into the pmiR-RB-REPORT ${ }^{\mathrm{TM}}$ luciferase reporter vectors (Guangzhou RiboBio Co., Ltd.).

Luciferase reporter assay. The plasmids containing the 3'UTR of GRP78, miR-495 mimics and NC sequences were transfected into 5-8F cells, using Lipofectamine 2000 reagent. The cells were incubated for $48 \mathrm{~h}$ after transfection, and the activity of firefly luciferase (hRluc) and the internal control (hluc) were detected using Dual-Glo Luciferase Assay system (Promega Corp., Madison, WI, USA).

Clonogenic survival assay. Radioresistance was determined by colony survival assay after irradiation. Briefly, the cells were plated in 6-well plates and exposed to a series of radiation doses (2-10 Gy), and were then cultured for 12 days. Subsequently, the surviving colonies (defined as a colony with $>50$ cells) were counted and the survival fraction was calculated as the number of colonies divided by the number of cells seeded and multiplied by the plating efficiency. Plating efficiency was calculated as colonies/10 cells. Three separate experiments were performed.

Cell growth analysis. Cells were plated in 24-well culture plates $\left(2.5 \times 10^{4} /\right.$ well $)$, and incubated for $24 \mathrm{~h}$. Subsequently, they were irradiated with radiation of $6 \mathrm{~Gy}$, and cell growth was monitored by counting the number of cells at various time intervals. Three independent experiments were carried out in triplicate.

Statistical analysis. Data were analyzed using SPSS software version 17.0 (SPSS, Inc., Chicago, IL, USA), and the results were expressed as the mean \pm standard deviation (SD). Comparisons were performed using the Student t-test or $\chi^{2}$ test. Spearman's and Pearson's analysis was performed for correlation analysis. A P-value $<0.05$ was considered to indicate a statistically significant difference.

\section{Results}

Analysis of GRP78 protein expression in NPC and chronic rhinitis tissues. GRP78 protein expression was assessed in tissue specimens by IHC. The ratio of positive GRP78 expression was significantly higher in NPC tissue samples $(70.7 \%)$, where 65 samples out of 92, exhibited positive expression 
A GRP78 expression

(strong positive)

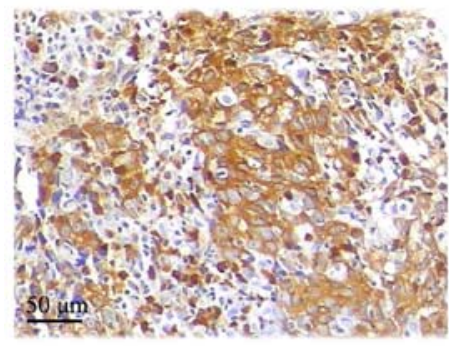

Radioresistant NPC tissue
B GRP78 expression (positive)

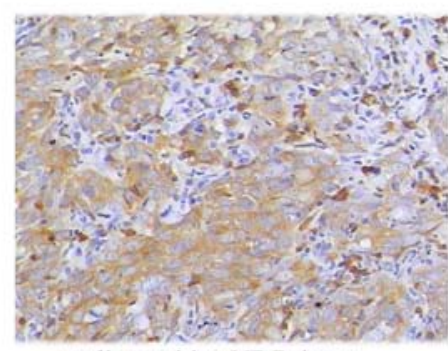

Radiosensitive NPC tissue
C $\quad$ GRP78 expression

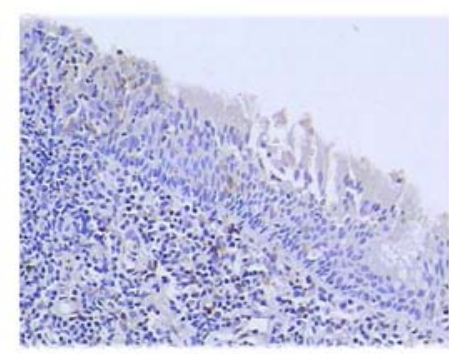

Chronic rhinitis tissue

Figure 1. Analysis of the GRP78 expression in NPC and chronic rhinitis tissues. (A) GRP78 expression in radioresistant NPC tissue (87.2\% strongly positive, 34/39). (B) GRP78 expression in radiosensitive NPC tissue (58.5\% positive, 31/53). (C) GRP78 expression in chronic rhinitis tissue (13.3\%, weakly positive/negative, 4/30). Amplification $\times 20$.

A

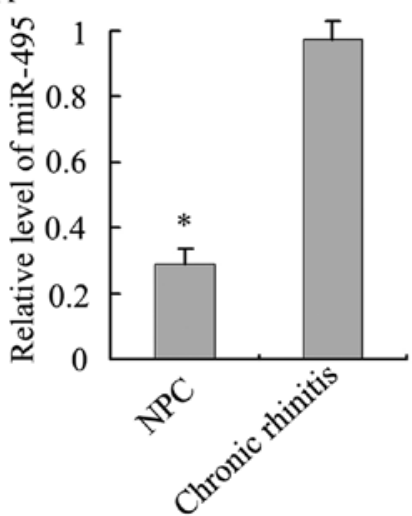

D

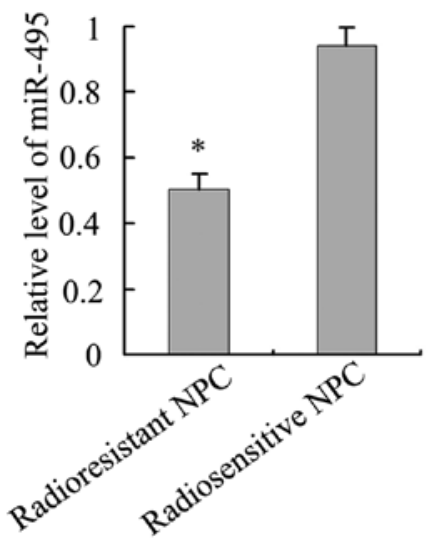

B

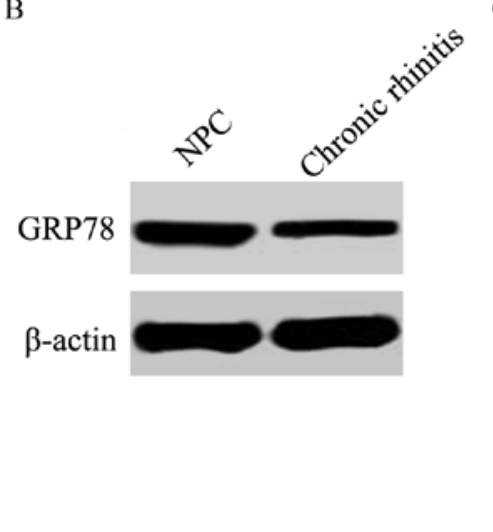

$\mathrm{E}$

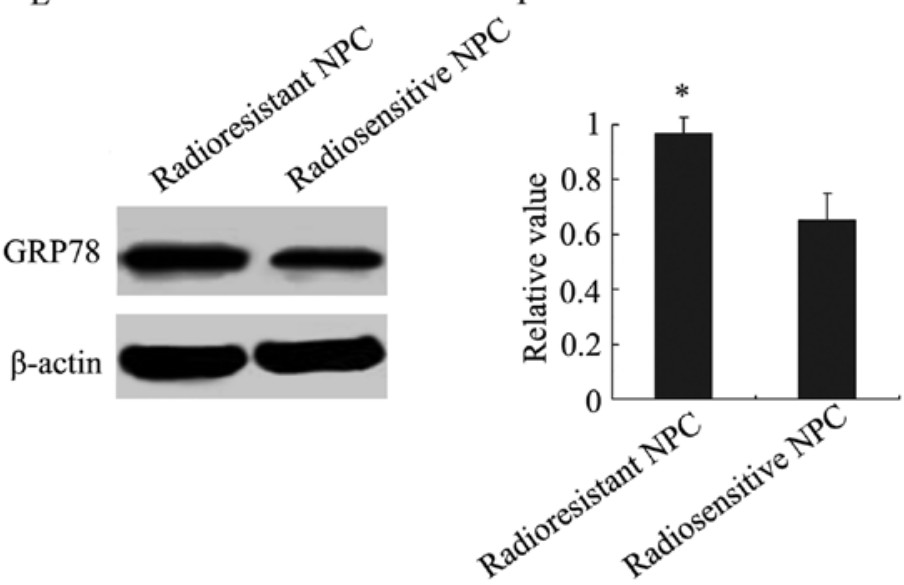

Figure 2. Analysis of miR-495 and GRP78 expression in NPC and chronic rhinitis tissues. (A) The relative level of miR-495 was lower in NPC tissue samples than in chronic rhinitis tissues. " $\mathrm{P}<0.05$, NPC vs. chronic rhinitis tissues. (B) The protein expression of GRP78 was higher in NPC and chronic rhinitis tissues. (C) The relative value of GRP78 normalized to $\beta$-actin expression in NPC and chronic rhinitis tissues. $\mathrm{P}<0.05$, NPC vs. chronic rhinitis tissues. (D) The relative level of miR-495 in radioresistant NPC was lower than in radiosensitive NPC tissue samples. ${ }^{*} \mathrm{P}<0.05$, radioresistant NPC vs. radiosensitive NPC. (E) The protein expression of GRP78 in radioresistant NPC was higher than in radiosensitive NPC tissue samples. (F) The relative value of GRP78 normalized to $\beta$-actin expression in radioresistant and radiosensitive NPC tissue samples. ${ }^{*} \mathrm{P}<0.05$, radioresistant NPC vs. radiosensitive NPC.

$(\mathrm{P}<0.05)$. However, in chronic rhinitis control tissue samples only $13.3 \%$ of the samples (4/30) exhibited positive expression. From further analysis of NPC tissue samples based on radiosensitivity information, we observed that $87.2 \%(34 / 39)$ of the radioresistant NPC patient samples significantly exhibited positive GRP78 staining, in comparison to $58.5 \%$ of the radiosensitive samples (31/53) (Fig. 1 and Table I).
Analysis of the relationship between miR-495 and GRP78 expression was then performed. We analyzed the expression of miR-495 in NPC and chronic rhinitis tissue samples, and our results demonstrated that its expression was lower in NPC tissue samples than chronic rhinitis tissues (Fig. 2A). Similarly, we also observed that miR-495 expression was lower in radioresistant tissue samples than radiosensitive 
A $5-8$ F +10 Gy Day 5

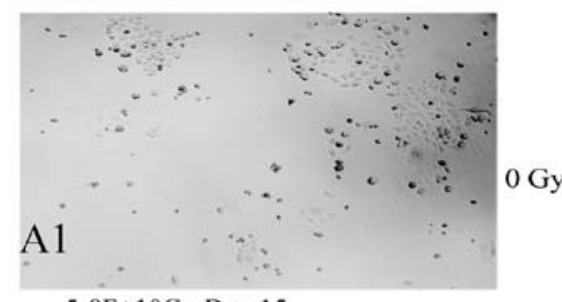

$5-8 \mathrm{~F}+10$ Gy Day 15

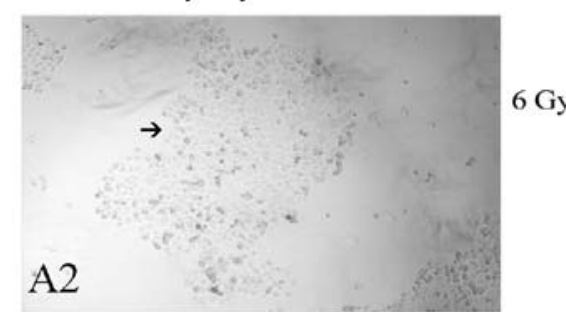

C

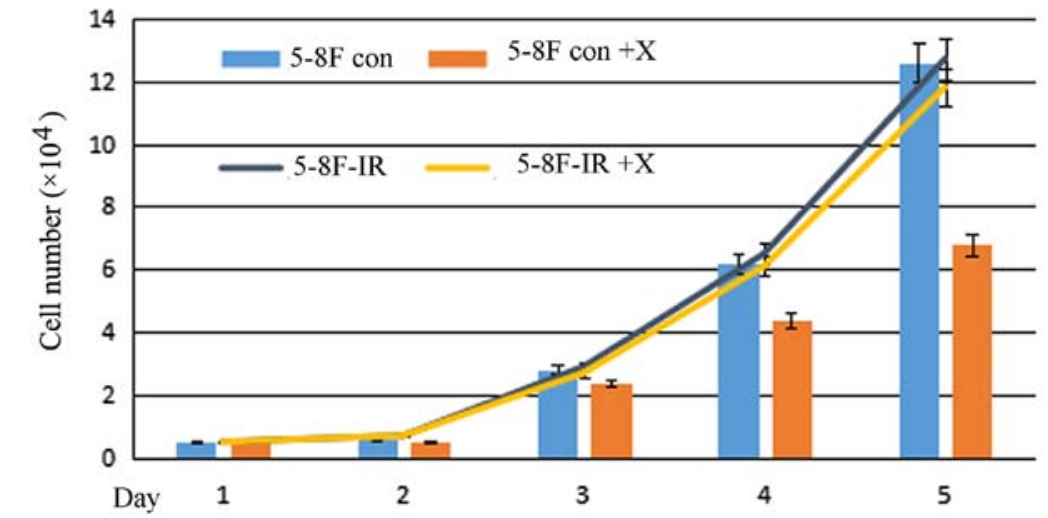

Figure 3. 5-8F-IR cells with radioresistance derived from irradiated 5-8F cells. (A-1) Only 5\% of the cells survived following irradiation of 5-8F cells with 10 Gy X-ray after 5 days (A-2) Fifteen days later a large subclone was selected, and named for 5-8F-IR (black arrow). (B and C) The subclone 5-8F-IR irradiated with 6 Gy (a medium dosage) X-ray was more resistant than the parent 5-8F.

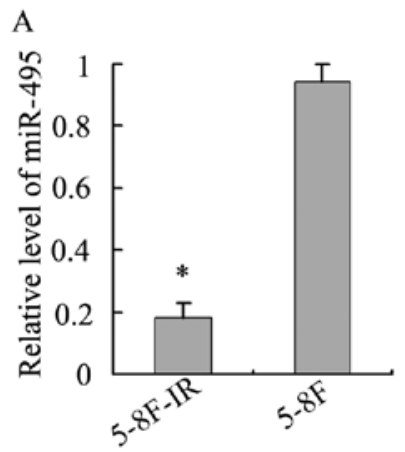

B

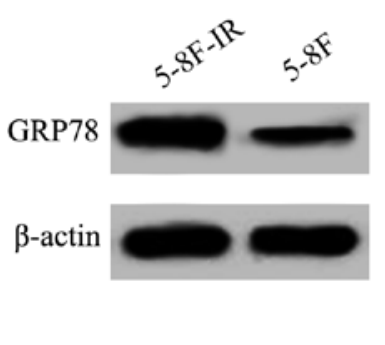

$\mathrm{C}$

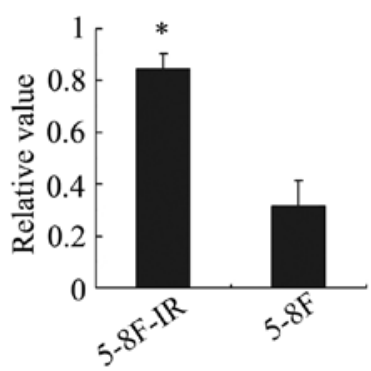

Figure 4. Analysis of miR-495 and GRP78 expression in NPC cell lines. (A) The relative expression level of miR-495 in 5-8F was higher than in 5-8F-IR. ${ }^{*} \mathrm{P}<0.05,5-8 \mathrm{~F}$ vs. 5-8F-IR. (B) The protein expression of GRP78 in 5-8F was lower than in 5-8F-IR. (C) The relative value of GRP78 normalized to $\beta$-actin expression. ${ }^{*} \mathrm{P}<0.05,5-8 \mathrm{~F}$ vs. $5-8 \mathrm{~F}-\mathrm{IR}$.

NPC tissue samples (Fig. 2D). Accordingly, GRP78 was highly expressed in NPC tissue samples, especially in radioresistant NPC (Fig. 2B, C, E and F). An inverse association between miR-495 and GRP78 expression was revealed using Spearman's and Pearson's correlation analysis.

5-8F-IR with radioresistance from the irradiated 5-8F cells. From the $5-8 \mathrm{~F}$ cells that were irradiated with 10 Gy X-ray only $5 \%$ of the cells survived. Fifteen days later, the 5\%-surviving cells formed subclones. A large subclone was selected and named 5-8F-IR. The subclone 5-8F-IR was more resistant than the parent 5-8F (Fig. 3). The 5-8F-IR subclone was cultured as a radioresistant sample.

In addition, we analyzed miR-495 and GRP78 expression in the 5-8F-IR and 5-8F NPC cells. The radioresistant 5-8F-IR cells exhibited lower expression of miR-495, in comparison to the parent $5-8 \mathrm{~F}$ cells, while the opposite was obtained for GRP78 ( $\mathrm{P}<0.05$; Fig. 4), and this further confirmed the 


\begin{tabular}{|c|c|c|c|c|c|c|c|c|c|c|c|c|c|}
\hline Gene Name & MicroRNA & StemLoop ID & DIANAmT & miRanda & miRDB & miRWalk & RNAhybrid & PICTAR4 & \begin{tabular}{|l|} 
PICTAR5 \\
\end{tabular} & $\mid$ PITA & RNA22 & Targetscan & sum \\
\hline HSPA5 & hsa-miR-30d & hsa-mir-30d & 1 & 1 & 0 & 1 & 1 & 1 & 1 & 0 & 0 & 1 & 7 \\
\hline HSPA5 & hsa-miR-199b-5p & hsa-mir-199b & 0 & 1 & 1 & 1 & 1 & 1 & 1 & 0 & 0 & 1 & 7 \\
\hline HSPA5 & hsa-miR-30c & hsa-mir-30c-1 & 1 & 1 & 0 & 1 & 1 & 1 & 1 & 0 & 0 & 1 & 7 \\
\hline HSPA5 & hsa-miR-30b & hsa-mir-30b & 1 & 1 & 0 & 1 & 1 & 1 & 1 & 0 & 0 & 1 & 7 \\
\hline HSPA5 & hsa-miR-338-5p & hsa-mir-338 & 1 & 1 & 1 & 1 & 0 & 0 & 1 & 0 & 0 & 1 & 6 \\
\hline HSPA5 & hsa-miR-199a-5p & hsa-mir-199a-2 & 1 & 1 & 1 & 1 & 0 & 0 & 1 & 0 & 0 & 1 & 6 \\
\hline HSPA5 & hsa-miR-329 & hsa-mir-329-2 & 1 & 1 & 1 & 1 & 0 & 0 & 1 & 0 & 0 & 1 & 6 \\
\hline HSPA5 & hsa-miR-378 & hsa-mir-378 & 1 & 1 & 1 & 1 & 0 & 0 & 1 & 0 & 0 & 1 & 6 \\
\hline HSPA5 & hsa-miR-635 & hsa-mir-635 & 1 & 1 & 1 & 1 & 0 & 0 & 1 & 0 & 0 & 1 & 6 \\
\hline HSPA5 & hsa-miR-597 & hsa-mir-597 & 1 & 1 & 1 & 1 & 0 & 0 & 1 & 0 & 0 & 1 & 6 \\
\hline HSPA5 & hsa-miR-422a & hsa-mir-422a & 1 & 1 & 1 & 1 & 0 & 0 & 1 & 0 & 0 & 1 & 6 \\
\hline HSPA5 & hsa-miR-605 & hsa-mir-605 & 1 & 1 & 1 & 1 & 0 & 0 & 1 & 0 & 0 & 1 & 6 \\
\hline HSPA5 & hsa-miR-495 & hsa-mir-495 & 1 & 1 & 1 & 1 & 0 & 0 & 1 & 0 & 0 & 1 & 6 \\
\hline HSPA5 & hsa-miR-145 & hsa-mir-145 & 1 & 1 & 0 & 1 & 1 & 0 & 1 & 0 & 0 & 1 & 6 \\
\hline HSPA5 & hsa-miR-619 & hsa-mir-619 & 1 & 1 & 1 & 1 & 0 & 0 & 1 & 0 & 0 & 1 & 6 \\
\hline HSPA5 & hsa-miR-590-3p & hsa-mir-590 & 1 & 1 & 1 & 1 & 0 & 0 & 1 & 0 & 0 & 1 & 6 \\
\hline HSPA5 & hsa-miR-30c & hsa-mir-30c-2 & 1 & 1 & 0 & 1 & 1 & 1 & 0 & 0 & 0 & 1 & 6 \\
\hline HSPA5 & hsa-miR-873 & hsa-mir-873 & 1 & 1 & 1 & 1 & 0 & 0 & 1 & 0 & 0 & 1 & 6 \\
\hline
\end{tabular}

Figure 5. Prediction of the interaction of miRNAs with GRP78 using miRWalk software. Eighteen different miRNAs that appear to interact with HSPA5 (Gene alias: GRP78) mRNA were predicted using the miRWalk program, which has the combined power of ten different programs. 0: Not predicted; 1: Predicted
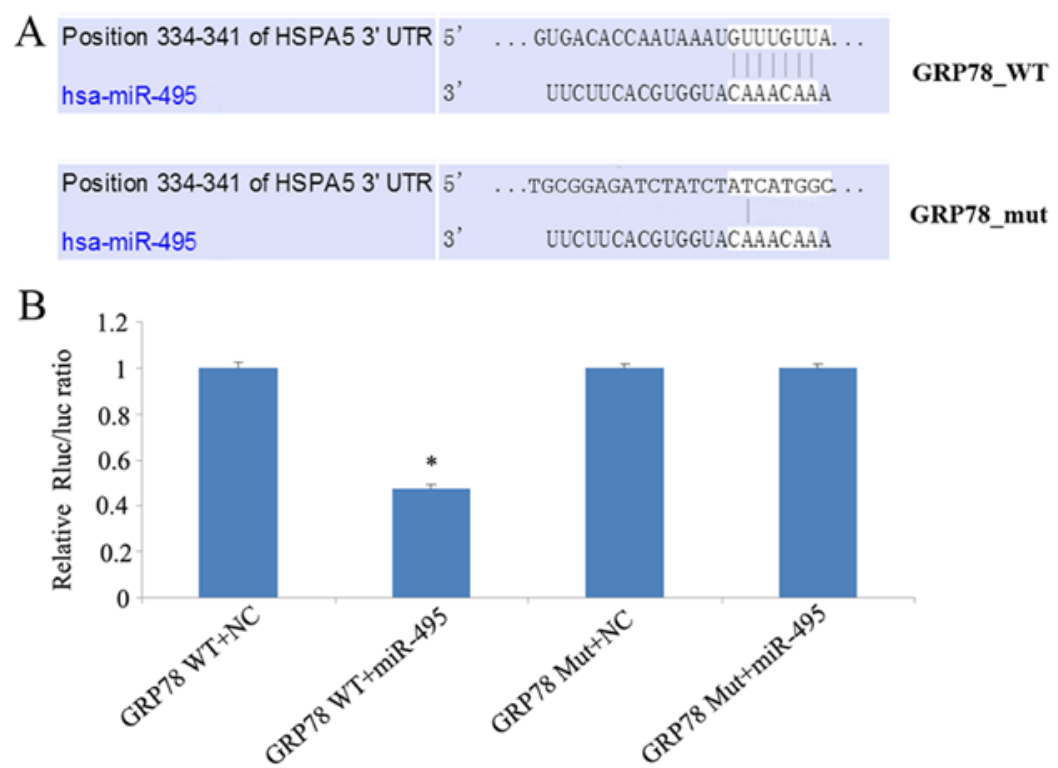

Figure 6. Analysis of the interaction between GRP78 and miR-495 by dual luciferase assay. (A) The miR-495 sequence was predicted to bind GRP78 (HSPA5) mRNA. (B) This luciferase assay revealed that miR-495 mimics could not target mutant GRP78, and could only reduce the expression of wild-type GRP78. miR-495 mimics significantly decreased the luciferase activity of WT GRP78-transfected cells, in comparison to cells transfected with its 3 'UTR mutant. "P<0.05, GRP78 WT+miR-495 vs. NC.

existence of an inverse relationship between the expression of miR-495 and GRP78.

Prediction of the interactions between miRNAs and GRP78 $m R N A$. Using the miRWalk program, which has the combined power of ten different programs, we predicted 18 different miRNAs that appeared to interact with HSPA5 (Gene alias: GRP78) mRNA (Fig. 5). Among these 18 predicted miRNAs, we further narrowed our search based on the highest binding scores for GRP78 mRNA, using miRanda software, which 

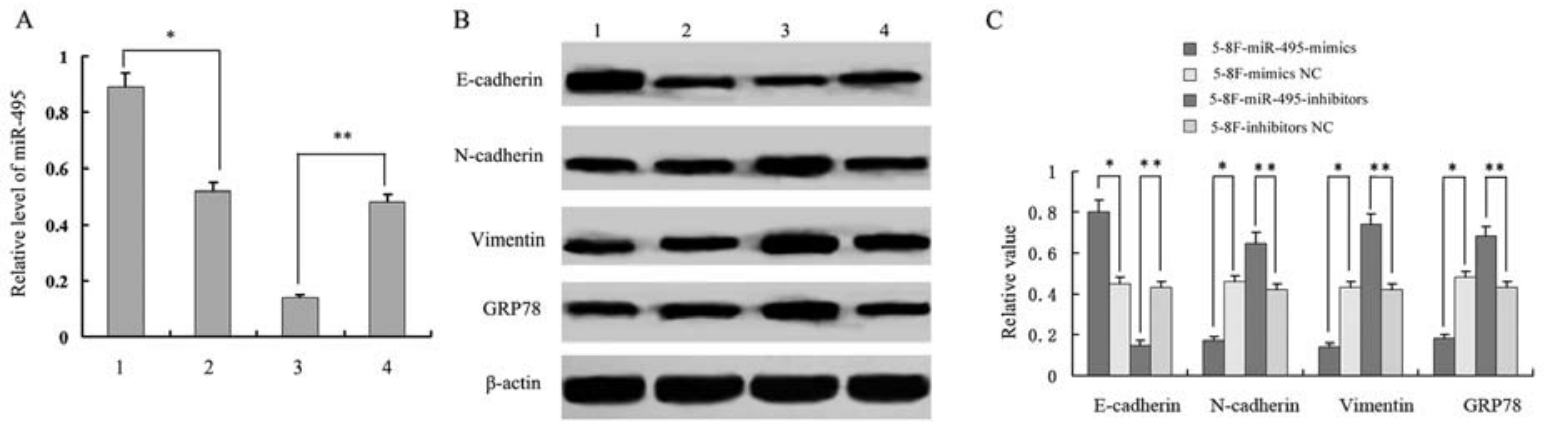

Figure 7. Expression analysis of the miR-495 and GRP78 and EMT-related proteins. (A) The relative miR-495 expression. (B and C) Western blot analysis and quantification of EMT-related proteins. Lane 1 represents 5-8F-miR-495-mimics; lane 2 represents, 5-8F-mimics NC; lane 3 represents 5-8F-miR-495-inhibitors; and lane 4 represents 5-8F-inhibitors NC. "P<0.05, 5-8F-miR-495-mimics vs. 5-8F-mimics NC; ${ }^{* *} \mathrm{P}<0.01,5-8 \mathrm{~F}-\mathrm{miR}$-495-inhibitors vs. 5-8F-inhibitors NC.

Table II. mirSVR and PhastCons scores of 18 predicted miRNAs.

mirSVR score Ranking PhastCons score

\begin{tabular}{lrrl}
\hline hsa-miR-495 & -1.2492 & 1 & 0.7212 \\
hsa-miR-199b-5p & -1.1437 & 2 & 0.7212 \\
hsa-miR-199a-5p & -1.1437 & 2 & 0.7212 \\
hsa-miR-597 & -1.1257 & 4 & 0.7212 \\
hsa-miR-338-5p & -1.0612 & 5 & 0.6955 \\
hsa-miR-873 & -0.8443 & 6 & 0.5165 \\
hsa-miR-590-3p & -0.8084 & 7 & 0.5061 \\
hsa-miR-605 & -0.7911 & 8 & 0.4909 \\
hsa-miR-635 & -0.6991 & 9 & 0.5104 \\
hsa-miR-619 & -0.6745 & 10 & 0.5165 \\
hsa-miR-378 & -0.3412 & 11 & 0.5165 \\
hsa-miR-422a & -0.3412 & 11 & 0.5165 \\
hsa-miR-329 & -0.1813 & 13 & 0.6127 \\
hsa-miR-30e & -0.1665 & 14 & 0.7104 \\
hsa-miR-30b & -0.1649 & 15 & 0.7104 \\
hsa-miR-30c & -0.1649 & 15 & 0.7104 \\
hsa-miR-30d & -0.1634 & 17 & 0.7104 \\
hsa-miR-145 & -0.0698 & 18 & 0.6840 \\
\hline
\end{tabular}

incorporates thermodynamic and sequence conservation scores. This analysis led to the identification of four miRNAs based on the following selection criteria (mirSVR $\leq-0.1$, phastCons 0.5-0.8) (Table II). Among these miRNAs, miR-495 was predicted to bind GRP78 mRNA with the highest stability and specificity.

Analysis of the mechanism of miR-495-mediated regulation of GRP78 expression. The inverse association between miR-495 and GRP78 expression in both NPC patient tissue samples and cell lines, led us to investigate whether miR-495 could influence GRP78 expression. We investigated whether miR-495 directly targeted the 3'UTR of GRP78, using a dual luciferase assay. In this regard, $5-8 \mathrm{~F}$ cells were co-transfected with either miR-495 mimics or corresponding negative control, along with a dual luciferase plasmid of wild-type (WT) or mutant (Mut) 3'UTR GRP78. This luciferase assay revealed that miR-495 mimics significantly decreased the luciferase activity of WT GRP78-transfected cells, in comparison to cells transfected with its 3'UTR mutant (Fig. 6). Overall our data revealed that miR-495 mimics could not target mutant GRP78, and could only reduce the expression of wild-type GRP78, thereby demonstrating that miR-495 targets the 3'UTR of GRP78.

Analysis of the relationship of $m i R-495$ and GRP78 expression with EMT-related proteins. In addition to downregulation of GRP78 expression by miR-495 mimics in 5-8F cells, we also observed the reduced expression of vimentin and $\mathrm{N}$-cadherin expression, and the increased expression of E-cadherin. This may indicate a EMT phenotype (reduced EMT). In parallel, when miR-495 expression was inhibited in 5-8F cells by treating them with miR-495 inhibitors, we observed an increased expression of GRP78, vimentin and N-cadherin, and a decreased expression of E-cadherin $(\mathrm{P}<0.05$, Fig. 7$)$. This may increase the EMT phenotype. These results indicated that miR-495 not only mediated the expression of GRP78, but was also involved in the expression of EMT-related proteins E-cadherin, $\mathrm{N}$-cadherin and vimentin. Following miR-495 treatment, 5-8F cells underwent morphological changes. The $5-8 \mathrm{~F}$ cells became rounder and the pseudopodiums became smaller with reduced EMT phenotype. Therefore, it was revealed that miR-495 influenced the EMT process (Fig. 8).

miR-495 upregulation enhances the efficacy of radiation therapy in NPC cells. Finally, we assessed whether regulation of miR-495 expression could influence the sensitivity of NPC cells against radiation therapy. The miR-495 mimics, inhibitors and additional negative control sequences were transfected into radioresistant $5-8 \mathrm{~F}$ cells $(5-8 \mathrm{~F}-\mathrm{IR})$, which were later irradiated with a range of radiation doses (2-10 Gy) for $24 \mathrm{~h}$. The analysis of cell viability by MTT assay revealed that the 5-8F-IR cells transfected with miR-495 mimics had significantly reduced viability in comparison to the negative control sequence-transfected cells, as observed in Table III. In contrast, the 5-8F-IR cells transfected with miR-495 inhibitors exhibited significantly increased viability compared to the NC-transfected cells $(\mathrm{P}<0.05$; Fig. 9 and Table III). 
Table III. Cell viability of transfected NPC cell lines.

\begin{tabular}{lcccr}
\hline & \multicolumn{4}{c}{ Survival fraction (\%) } \\
\cline { 2 - 5 } Radiation doses (Gy) & miR-495 mimics-5-8F-IR & NC-5-8F-IR & miR-495 inhibitors-5-8F & NC-5-8F \\
\hline 2 & $92 \pm 2.0$ & $96 \pm 2.2$ & $97 \pm 2.0$ & $95 \pm 1.8$ \\
4 & $76 \pm 3.0$ & $88 \pm 3.0$ & $90 \pm 2.8$ & $82 \pm 2.8$ \\
6 & $58 \pm 2.8$ & $78 \pm 3.1$ & $82 \pm 3.2$ & $68 \pm 3.0$ \\
8 & $36 \pm 2.6$ & $68 \pm 2.4$ & $72 \pm 2.6$ & $52 \pm 3.1$ \\
10 & $16 \pm 2.8$ & $54 \pm 2.5$ & $60 \pm 3.1$ & $36 \pm 3.0$ \\
\hline
\end{tabular}

$\mathrm{RPF}_{1}=1.04 ; \mathrm{P}<0.05$

$\mathrm{RPF}_{2}=1.02 ; \mathrm{P}<0.05$

${ }^{\mathrm{a} P P F} F_{1}$ is the RPF of miR-495 mimics-5-8F-IR; $\mathrm{RPF}_{2}$ is the RPF of miR-495 inhibitors-5-8F. NPC, nasopharyngeal carcinoma. NC, negative control; IR, radioresistance.
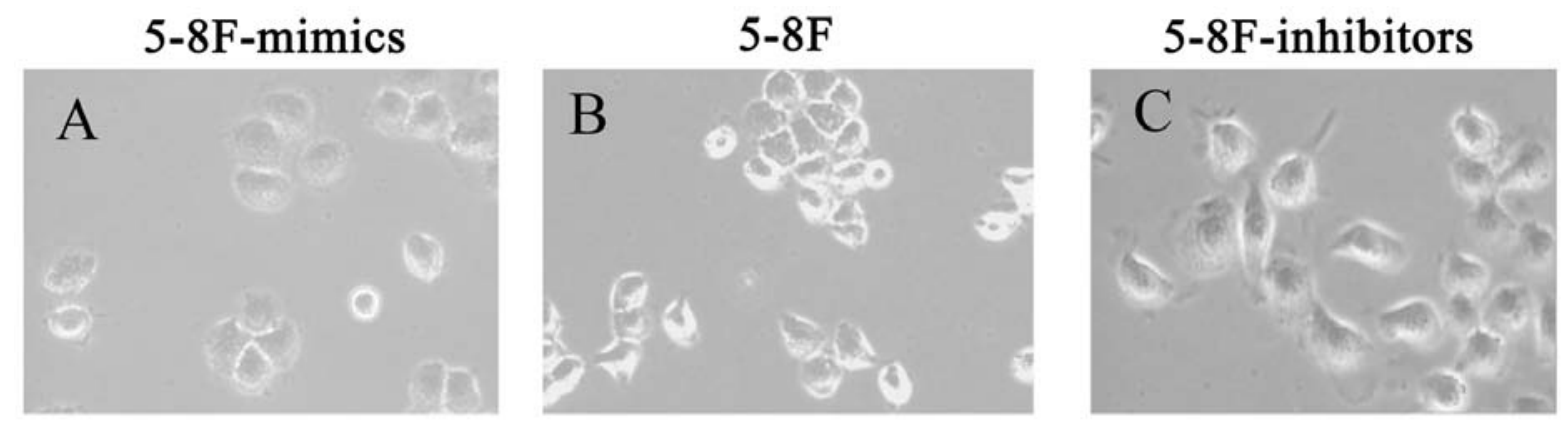

Figure 8. 5-8F cells with morphological changes following miR-495 treatment. Following miR-495 treatment, 5-8F cells exhibited morphological changes: (A) $5-8 \mathrm{~F}$ cells (oblate spheres) after miR-495-mimic transfection. (B) 5-8F control cells (small spheres). (C) 5-8F cells after miR-495-inhibitor transfection (large spheres).

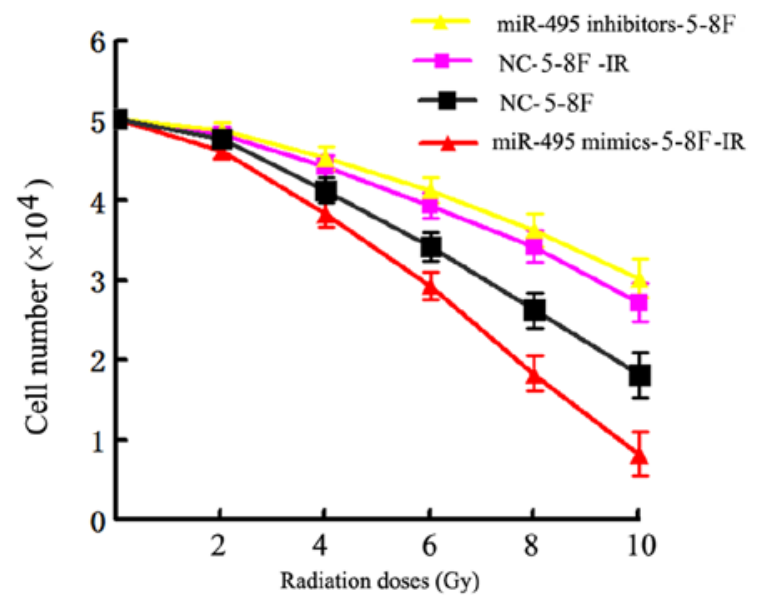

Figure 9. Analysis of the viability of NPC cell lines after being irradiated with a range of radiation doses (2-10 Gy). 5-8F-IR cells transfected with miR-495 mimics had significantly reduced viability in comparison to negative control sequence-transfected cells. The 5-8F-IR cells transfected with miR-495 inhibitors exhibited significantly increased viability as compared to the NC-transfected cells.

\section{Discussion}

The glucose-regulated protein GRP78, an endoplasmic reticulum stress-induced molecular chaperone, plays an important role in endoplasmic reticulum (ER) stress and in maintaining cellular homeostasis as a vital stress response survival protein $(15,16)$. Its expression is induced in cancer cells not only as a result of ER stress, due to glucose deprivation, severe hypoxia and acidosis, but also because of chemotherapeutic drugs and radiation therapy. It has been revealed to confer tumor resistance to chemotherapeutic agents and radiation therapy $(17,18)$. The GRP78 expression and distribution changes have been observed in multiple instances, including thrombotic disease and cancer $(19,20)$. It had been demonstrated that GRP78 is highly expressed in many tumors, thereby suggesting that it may play a vital role in tumor biology (21). In our previous study, we reported that GRP78 may act as radioresistant factor in NPC (4). In the present study based on IHC analysis, we established that NPC patient tissue samples exhibited significantly higher positive GRP78 staining, in comparison to chronic rhinitis patient samples. Specifically, a higher percentage of GRP78 positive staining was mostly observed in radioresistant NPC patient samples than radiosensitive NPC patient samples. These observations revealed that GRP78 may play a vital role in the progression of NPC and may act as a radioresistant agent in NPC.

To further shed light on the regulation of GRP78 expression in NPC, we performed bioinformatics analysis to identify miRNAs targeting GRP78, using miRWalk software. Typically, 
the interaction between miRNAs and mRNAs can occur through complementary binding of the base pairs. However, the interaction is not usually fully complementary, and several software programs have been developed to accurately predict miRNA-mRNA binding and to find specific target genes of miRNAs (22). Programs use algorithms to predict interaction, and the prediction results are not completely consistent between different programs. Thus, many researchers use a combination of predictive programs for definitive analysis. Among these miRWalk is an effective online software tool for predicting miRNA-mRNA binding, and is based on ten separate programs that help to predict results with more accuracy and credibility (23). In the present study, using this software, we predicted 18 miRNAs that could interact with GRP78 mRNA. Among them, the interaction of miR-495 was predicted to have the highest stability and specificity, indicating its preferential binding to GRP78 mRNA.

Notably, the parallel analysis of miR-495 expression in NPC and chronic rhinitis tissue samples revealed an inverse association with GRP78 expression. In addition, miR-495 expression was observed to be lower in radioresistant NPC tissues, than in radiosensitive NPC tissue samples. Similarly, radioresistant 5-8F-IR cells also displayed lower expression of miR-495 than radiosensitive parental 5-8F cells. In contrast, GRP78 was higher in radioresistant 5-8F-IR cells, while low in radiosensitive parental $5-8 \mathrm{~F}$ cells. These observations led us to hypothesize that GRP78 may be a target of miR-495, and may play a role in NPC radioresistance.

Ahmadi et al (24) found that miR-495 and miR-199-5p could bind to the 3'-UTR of GRP78 in non-small cell lung cancer (NSCLC) and miR-495 may play a causative role in tumorigenesis of lung cancer. Their results revealed upregulation of GRP78 and a concomitant downregulation of miR-495 and miR-199a-5p in NSCLC, which was consistant with our study on NPC. Based on the findings of Ahmadi et al and our primary study of GRP78 which revealed its association with NPC radioresistance, this study emphasized and elucidated the mechanism of mediation of NPC radioresistance by miR-495 targeting GRP78 in more depth. To further explore our hypothesis, we directly explored the relationship between miR-495 and GRP78 by undertaking a dual luciferase assay, where miR-495 mimics were transfected into 5-8F cells along with either wild-type GRP78 or 3'UTR mutant, both cloned into luciferase vectors. Our experiments revealed that miR-495 mimics were not capable of targeting mutant GRP78, while they targeted wild-type GRP78 and led to decreased GRP78 expression. Therefore, our study concluded that miR-495 targeted the 3'UTR of GRP78.

Recently some studies have suggested a link between EMT and radioresistance. A study by Jong et al (25) indicated that EMT and low expression of EMT-inhibiting miRNAs (especially miR203A), were observed in pretreatment samples, and caused intrinsic radioresistance in head and neck squamous cell carcinoma (HNSCC). Thus EMT, a complex and dynamic process and one of the early events in remodeling of the cytoskeleton, is characterized by increased expression of mesenchymal markers, such as N-cadherin and vimentin, and decreased expression of epithelial markers, such as E-cadherin $(26,27)$. In addition, multiple studies have shown that EMT plays a vital role in the occurrence, development, invasion and metastasis of tumors $(28,29)$. Thus, in the present study, we observed that upregulation of miR-495 expression in 5-8F cells after miR-495-mimic transfection, resulted in downregulation of GRP78, vimentin and $\mathrm{N}$-cadherin expression and upregulation of E-cadherin expression. Conversely, downregulation of miR-495 in 5-8F cells by its inhibitor reversed the expression trends of GRP78 and EMT proteins. These data demonstrated that miR-495 not only targets GRP78 but may also be involved in the regulation of EMT markers.

Finally, by assessing the effects of miR-495 mimics on post-exposure with different doses of radiation it was observed that the viability of 5-8F-IR cells was significantly decreased. In contrast, miR-495 inhibitors significantly enhanced 5-8F cell viability. These experiments indicated that miR-495 may regulate radiation sensitivity of NPC cells.

In conclusion, the present study revealed that the GRP78 protein was highly expressed in NPC than in chronic rhinitis patient tissue samples. In addition, the enhanced expression of GRP78 was associated with radioresistant NPC samples. Bioinformatics analysis indicated that miR-495 interacted strongly with GRP78 and notably followed an inverse expression trend. Additional studies suggested that miR-495 not only reduced GRP78 expression by targeting its 3'UTR, but may also be involved in the expression of EMT-related proteins. Elucidation of the influence of miR-495-targeting GRP78 on EMT and a knockdown assay of GRP78, which are limitations of the present study, need to be performed in a future study. Finally, we also observed that miR-495 mimics sensitized NPC cells against various doses of radiation. However, further studies are required to ascertain the significance and mechanism of miR-495-mediated regulation of EMT, and the subsequent contributions to radiation sensitivity in NPC cells.

\section{Acknowledgements}

Not applicable.

\section{Funding}

The present study was supported by the National Natural Science Foundation of China (81372905), the Hunan Provincial Natural Science Foundation of China (2015JJ4075) and the Hunan Provincial Health and Family Planning Commission, China (no. C2017044).

\section{Availability of data and materials}

The data sets used during the present study are available from the corresponding author upon reasonable request.

\section{Authors' contributions}

XF and QH planned the study. XF, WL, SW and QH performed the experiments and data analysis. QH wrote the study with input from XF, WL and SW. All authors read and approved the manuscript and agree to be accountable for all aspects of the research in ensuring that the accuracy or integrity of any part of the work are appropriately investigated and resolved. 


\section{Ethics approval and consent to participate}

NPC tissue biopsies (pre-therapy) were obtained after receiving informed consent from all patients and were used for immunohistochemical (IHC) staining. This study was certified by the Ethics Committee of Xiangya School of Medicine (Central South University, China).

\section{Patient consent for publication}

Not applicable.

\section{Competing interests}

The authors declare that they have no competing interests.

\section{References}

1. Chen $\mathrm{W}$ and $\mathrm{Hu} \mathrm{GH}$ : Biomarkers for enhancing the radiosensitivity of nasopharyngeal carcinoma. Cancer Biol Me 12: 23-32, 2015.

2. Yi HM, Yi H, Zhu JF, Xiao T, Lu SS, Guan YJ and Xiao ZQ: A five-variable signature predicts radioresistance and prognosis in nasopharyngeal carcinoma patients receiving radical radiotherapy. Tumour Biol 37: 2941-2949, 2016.

3. Yu YH, Xia WX, Shi JL, Ma WJ, Li Y, Ye YF, Liang H, Ke LR, Lv X, Yang J, et al: A model to predict the risk of lethal nasopharyngeal necrosis after re-irradiation with intensity-modulated radiotherapy in nasopharyngeal carcinoma patients. Chin J Cancer 35: 59, 2016.

4. Feng XP, Yi H, Li MY, Li XH, Yi B, Zhang PF, Li C, Peng F, Tang CE, Li JL, et al: Identification of biomarkers for predicting nasopharyngeal carcinoma response to radiotherapy by proteomics. Cancer Res 70: 3450-3462, 2010.

5. Tian S, Chang W, Du H, Bai J, Sun Z, Zhang Q, Wang H, Zhu G, Tao K and Long Y: The interplay between GRP78 expression and Akt activation in human colon cancer cells under celecoxib treatment. Anticancer Drugs 26: 964-973, 2015.

6. Cook KL, Soto-Pantoja DR, Clarke PA, Cruz MI, Zwart A, Wärri A, Hilakivi-Clarke L, Roberts DD and Clarke R Endoplasmic reticulum stress protein GRP78 modulates lipid metabolism to control drug sensitivity and antitumor immunity in breast cancer. Cancer Res 76: 5657-5670, 2016.

7. Zhao S, Li H, Wang Q, Su C, Wang G, Song H, Zhao L, Luan Z and Su R: The role of $\mathrm{c}$-Src in the invasion and metastasis of hepatocellular carcinoma cells induced by association of cell surface GRP78 with activated $\alpha 2 \mathrm{M}$. BMC Cancer 15: 389, 2015.

8. Cook KL and Clarke R: Role of GRP78 in promoting therapeuticresistant breast cancer. Future Med Chem 7: 1529-1534, 2015.

9. Yu T, Guo Z, Fan H, Song J, Liu Y, Gao Z and Wang Q: Cancer-associated fibroblasts promote non-small cell lung cancer cell invasion by upregulation of glucose-regulated protein 78 (GRP78) expression in an integrated bionic microfluidic device. Oncotarget 7: 25593-25603, 2016.

10. Li C, Zhang B, Lv W, Lai C, Chen Z, Wang R, Long X and Feng X: Triptolide inhibits cell growth and GRP78 protein expression but induces cell apoptosis in original and radioresistant NPC cells. Oncotarget 7: 49588-49596, 2016

11. He L and Hannon GJ: MicroRNAs: Small RNAs with a big role in gene regulation. Nat Rev Genet 5: 522-531, 2004.

12. Bartel DP: MicroRNAs: Target recognition and regulatory functions. Cell 136: 215-233, 2009.

13. To EW, Chan KC, Leung SF, Chan LY, To KF, Chan AT, Johnson PJ and Lo YM: Rapid clearance of plasma Epstein-Barr virus DNA after surgical treatment of nasopharyngeal carcinoma. Clin Cancer Res 9: 3254-3259, 2003.
14. Shanmugaratnam K and Sobin LH: The World Health Organization histological classification of tumours of the upper respiratory tract and ear. A commentary on the second edition. Cancer 71: 2689-2697, 1993.

15. Flodby P, Li C, Liu Y, Wang H, Marconett CN, Laird-Offringa IA, Minoo P, Lee AS and Zhou B: The 78-kD Glucose-regulated protein regulates endoplasmic reticulum homeostasis and distal epithelial cell survival during lung development. Am J Respir Cell Mol Biol 55: 135-149, 2016.

16. Kureel J, John AA, Raghuvanshi A, Awasthi P, Goel A and Singh D: Identification of GRP78 as a molecular target of medicarpin in osteoblast cells by proteomics. Mol Cell Biochem 418: 71-80, 2016.

17. Kuang XY, Jiang HS, Li K, Zheng YZ, Liu YR, Qiao F, Li S, $\mathrm{Hu} \mathrm{X}$ and Shao ZM: The phosphorylation-specific association of STMN1 with GRP78 promotes breast cancer metastasis. Cancer Lett 377: 87-96, 2016

18. Gifford JB, Huang W, Zeleniak AE, Hindoyan A, Wu H, Donahue TR and Hill R: Expression of GRP78, master regulator of the unfolded protein response, increases chemoresistance in pancreatic ductal adenocarcinoma. Mol Cancer Ther 15: 1043-1052, 2016

19. Zhang B, Han H, Fu S, Yang P, Gu Z, Zhou Q and Cao Z: Dehydroeffusol inhibits gastric cancer cell growth and tumorigenicity by selectively inducing tumor-suppressive endoplasmic reticulum stress and a moderate apoptosis. Biochem Pharmacol 104: 8-18, 2016.

20. Kaira K, Toyoda M, Shimizu A, Imai H, Sakakura K, Nikkuni O, Suzuki M, Iijima M, Asao T and Chikamatsu K: Decreasing expression of glucose-regulated protein GRP78/BiP as a significant prognostic predictor in patients with advanced laryngeal squamous cell carcinoma. Head Neck 38: 1539-1544, 2016.

21. Nami B, Ghasemi-Dizgah A and Vaseghi A: Overexpression of molecular chaperons GRP78 and GRP94 in CD $44^{\text {hi }} / C D 24^{\text {lo }}$ breast cancer stem cells. Bioimpacts 6: 105-110, 2016.

22. Dweep H, Sticht C, Pandey P and Gretz N: miRWalk-database: Prediction of possible miRNA binding sites by 'walking' the genes of three genomes. J Biomed Inform 44: 839-847, 2011.

23. Dweep H, Gretz N and Sticht C: miRWalk database for miRNA-target interactions. Methods Mol Biol 1182: 289-305, 2014.

24. Ahmadi A, Khansarinejad B, Hosseinkhani S, Ghanei M and Mowla SJ: miR-199a-5p and miR-495 target GRP78 within UPR pathway of lung cancer. Gene 620: 15-22, 2017.

25. de Jong MC, Ten Hoeve JJ, Grénman R, Wessels LF, Kerkhoven R, Te Riele H, van den Brekel MW, Verheij M and Begg AC: Pretreatment microRNA expression impacting on Epithelial-to-mesenchymal transition predicts intrinsic radiosensitivity in head and neck cancer cell lines and patients. Clin Cancer Res 21: 5630-5638, 2015.

26. Shenoy AK, Jin Y,LuoH, Tang M,Pampo C, Shao R, Siemann DW, Wu L, Heldermon CD, Law BK, et al: Epithelial-to-mesenchymal transition confers pericyte properties on cancer cells. J Clin Invest 126: 4174-4186, 2016.

27. Nishitani S, Noma K, Ohara T, Tomono Y, Watanabe S, Tazawa H, Shirakawa Y and Fujiwara T: Iron depletion-induced downregulation of $\mathrm{N}$-cadherin expression inhibits invasive malignant phenotypes in human esophageal cancer. Int $\mathbf{J}$ Oncol 49: 1351-1359, 2016.

28. Zhang Z, Bu X, Chen H, Wang Q and Sha W: Bmi-1 promotes the invasion and migration of colon cancer stem cells through the downregulation of E-cadherin. Int J Mol Med 38: 1199-1207, 2016.

29. Petrova YI, Schecterson L and Gumbiner BM: Roles for E-cadherin cell surface regulation in cancer. Mol Biol Cell 27: 3233-3244, 2016.

This work is licensed under a Creative Commons Attribution-NonCommercial-NoDerivatives 4.0 International (CC BY-NC-ND 4.0) License. 